
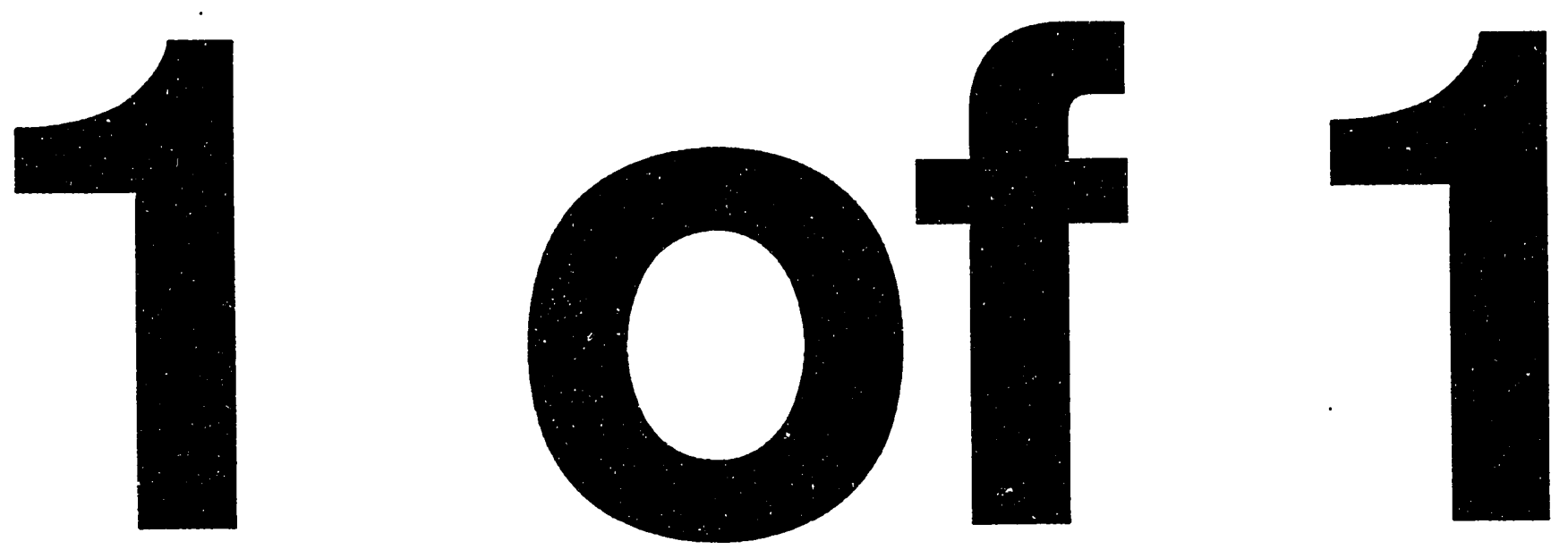
ANL/RE-93/9

\section{Argonne National Laboratory \\ 9700 South Cass Avenue \\ Argonne, Illinois 60439}

\section{SLOSHING RESPONSE OF NONUNIFORM DENSITY LIQUID IN A LATERALLY EXCITED TANK}

by

Yu Tang and Yao W. Chang

Reactor Engineering Division

October 1993

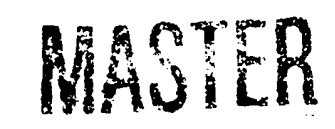

DISTRIBUTION OF THIS DOCUMENT IS UNLIMITED

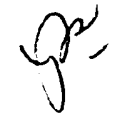




\section{TABLE OF CONTENTS}

Page

ABSTRACT $\ldots \ldots \ldots \ldots \ldots \ldots \ldots \ldots \ldots \ldots \ldots \ldots \ldots \ldots \ldots \ldots \ldots \ldots$

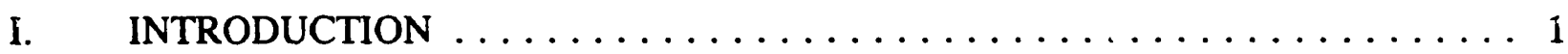

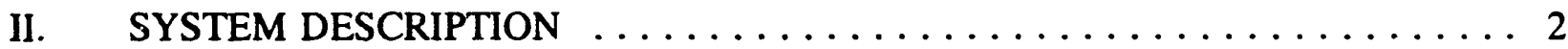

III. APPROACH AND SOLUTIONS $\ldots \ldots \ldots \ldots \ldots \ldots \ldots \ldots \ldots \ldots \ldots \ldots$

A. Impulsive Component $\ldots \ldots \ldots \ldots \ldots \ldots \ldots \ldots \ldots \ldots$

B. Convective Component $\ldots \ldots \ldots \ldots \ldots \ldots \ldots \ldots \ldots \ldots$

IV. NUMERICAL RESULTS $\ldots \ldots \ldots \ldots \ldots \ldots \ldots \ldots \ldots \ldots \ldots$

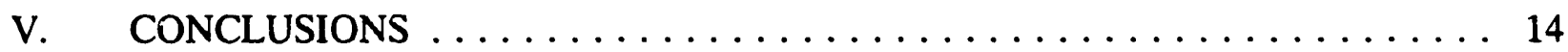

ACKNOWLEDGMENT $\ldots \ldots \ldots \ldots \ldots \ldots \ldots \ldots \ldots \ldots \ldots \ldots \ldots \ldots$

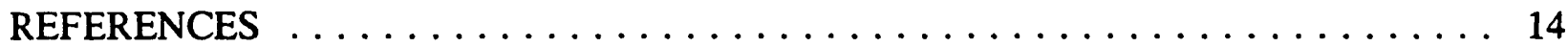




\section{LIST GF TABLES}

No.

Title

Page

I Convergence Table of Sloshing Frequency Coefficient, $\alpha_{1} \ldots \ldots \ldots \ldots$

II Convergence Table of Sloshing Displacement Coefficient, $r_{1} \ldots \ldots \ldots \ldots$

III Comparison of Response Quantities of the Tank-Liquid Systems Considered in Numerical Example $\ldots \ldots \ldots \ldots \ldots \ldots \ldots \ldots \ldots \ldots \ldots$

\section{LIST OF FIGURES}

No.

Title

Page

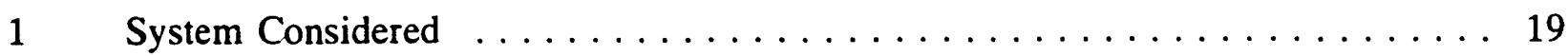

2 Mathematical Model for the Liquid Field $\ldots \ldots \ldots \ldots \ldots \ldots \ldots \ldots$

3 Effect of Liquid Density on the Sloshing Frequency $\ldots \ldots \ldots \ldots \ldots \ldots$

$4 \quad$ Effect of Liquid Density on the Sloshing Displacement $\ldots \ldots \ldots \ldots \ldots \ldots 22$

5 Convective Pressure Exerted on Wall of Rigid Tanks with $\mathrm{H} / \mathrm{R}=0.5 \ldots \ldots \ldots 23$

6 Convective Pressure Exerted on Wall of Rigid Tanks with $\mathrm{H} / \mathrm{R}=3 \ldots \ldots \ldots 23$ 


\title{
SLOSHING RESPONSE OF NONUNIFORM DENSITY \\ LIQUID IN A LATERALLY EXCITED TANK
}

by

\author{
Yu Tang and Yao W. Chang
}

\begin{abstract}
A study on the sloshing response of nonuniform density liquid in a tank undergoing lateral buse excitations is presented. The system considered is a circular cylindrical tank containing a liquid whose density increases with the liquid depth. The density distribution along the depth can be of any arbitrary continuous function. In the analysis, the liquid field is divided into $\mathbf{n}$ layers. The thicknesses of the liquid layers can be different, but the density of each liquid layer is considered to be uniform and its value is assigned to be the value of the original liquid density at the mid-height of that layei. The problem is solved by the transfer matrix technique. The effect of the nonuniform liquid density on the sloshing response is illustrated in a numerical example in which the linear and cosirie distributions of the liquid density are assumed. The response functions examined include the: sloshing frequencies, surface wave height, and the associated convective hydrodynamic pres'sure. The results are presented in tabular and graphical forms. It is found that the natural frequencies of the sloshing motion for nonuniform density liquid are lower than those of the uniform density liquid of the same total depth contained in an identical tank. Also, it is shown that for nonuniform density liquid, the maximum sloshing wave height may increase significantly and the magnitude of the convective hydrodynamic pressure may be quite different compared with that of a uniform liquid contained in an identical tank.
\end{abstract}




\section{INTRODUCTION}

The sloshing motion associated with the dynamic response of a homogeneous liquid in a rigid tank undergoing lateral base motion has been studied by Housner [1], Bauer [2] and Yang [3]. It is found that the natural frequencies of the sloshing motion can be computed by a very simple closed-form equation, and these frequencies are independent of the liquid density. For more information about this topic, the reader is referred to the above mentioned references. However, these previous studies were based on the assumption that the liquid has a constant density. Recently a paper by Bandyopadhyay [4] indicates that a large number of high level waste (HLW) storage tanks at various U.S. Department of Energy (DOE) facilities contain liquid with nonuniform density. Thus, there is a need to understand the effect of nonuniform density on the seismic response of the contained liquid. Exploratory studies on the dynamic response of tanks containing two liquids have been performed by Tang $[5,6]$. Those studies show that the dynamic response of a tank containing two liquids is quite different from that of an identical tank containing only one liquid. Especially, the sloshing wave height may increase significantly in a tank that contains two liquids. Therefore, to design and evaluate the HLW storage tanks it is necessary to understand the sloshing response of tanks that contain liquid with nonuniform density.

The objectives of this study are (1) to develop a method of analysis for the computation of the sloshing frequencies, the sloshing displacement and the convective hydrodynamic pressure of nonuniform density liquid contained in a tar : idergoing lateral base excitations; and (2) to examine the results of analysis from which the effect of nonuniform density of the liquid on the sloshing response can be evaluated. In the analysis, the liquid field is divided into $\mathrm{n}$ layers along its height. These liquid layers may have different thicknesses, but the liquid density of each layer is assumed to be uniform. The tank wall is assumed to be rigid. For each layer the so-called impulsive component of the response is solved first and then followed by the convective component of the response quantity. For the definitions of impulsive and convective components, the reader is referred to Refs. 1 and 7. Response functions examined include the sloshing frequencies, sloshing wave height and the convective hydrodynamic pressure. Even though, the 
solutions presented herein are for rigid tanks, they are also applicable for flexible tanks because it has been shown [8] that the sioshing motion is insensitive to the flexibility of the tank wall.

\section{SYSTEM DESCRIPTION}

The tank-liquid system investigated is shown in Fig. 1. It is a ground-supported upright circular cylindrical tank of radius $\mathrm{R}$ which is filled with a nonuniform liquid to a height of $\mathrm{H}$. The density of the liquid is assumed to have a minimum value, denoted by $\rho_{v}$, at the top of the liquid surface. The liquid density is assumed to increase monotonical with the increase of the liquid depth and reaches a maximum value, denoted by $\rho_{b}$, at bottom of the liquid. The tank is assumed to be rigid and clamped to a rigid base. The liquid is considered to be incompressible and inviscid. The response of the liquid is assumed to be linear. The cylindrical coordinate system, $r, \theta$, and $z$, is employed for the study with the origin defined at the center of the tank base where $\theta=0$ is assumed to be the direction of the seismic excitation. The lateral excitation considered herein is denoted by $\ddot{x}(t)$. The temporal variation of $\ddot{x}(t)$ can be arbitrary.

\section{APPROACH AND SOLUTIONS}

The liquid field is first divided into $\mathrm{n}$ layers as shown in Fig. 2. The thickness of the Layer $j(j=1,2,3 \ldots n)$ is denoted by $H_{j}$. The thicknesses of the different layers need not be the same. The liquid density for layer $\mathrm{H}_{j}$ is taken to be the value of the original liquid density at mid-height of the layer, and it is denoted by $\rho_{j}$. It is assumed that the liquid is uniform in each layer. Thus, the mathematical model that represents the physical system depicted in Fig. 1 has $\mathbf{n}$ layers of liquids with different thicknesses and liquid densities. For the convenience of derivation, a local cylindrical coordinate system, $r, \theta, z_{j}$, is introduced for the Layer $j$ where $z_{j}$ is related to $z$ by the equation 


$$
z=z_{j}+\sum_{k=1}^{j-1} H_{k} \text { for } 0 \leq z_{j} \leq H_{j}
$$

Given the conditions that the liquids are incompressible and inviscid, the hydrodynamic pressure induced at Layer $j$, densted by $p_{j}$, must satisfy the Laplace equation

$$
\nabla^{2} p_{j}=C \text { for } \mathrm{j}=1,2,3 \ldots \mathrm{n}
$$

in the region $0 \leq \mathrm{r} \leq \mathrm{R}, 0 \leq \theta \leq 2 \pi$, and $0 \leq \mathrm{z}_{\mathrm{j}} \leq \mathrm{H}_{\mathrm{j}}$. The liquid acceleration at an arbitrary point in Layer $\mathrm{j}$ along s-direction is given by

$$
a_{s}=-\frac{1}{\rho_{j}} \frac{\partial p_{j}}{\partial s}
$$

The boundary conditions are:

1. The vertical acceleration of liquid at the tank base must be zero; i.e.,

$$
\left.\frac{\partial p_{1}}{\partial z_{1}}\right|_{z_{1}=0}=0
$$

2. The radial acceleration of liquid adjacent to the tank wall must equal the acceleration of the tank wall, i.e.,

$$
-\left.\frac{1}{\rho_{j}} \frac{\partial p_{j}}{\partial r}\right|_{r=R}=\ddot{x}(t) \cos \theta, \quad j=1,2 \ldots n
$$

3. At the free surface, the linearized boundary condition is

$$
\left(\frac{\partial^{2} p_{n}}{\partial t^{2}}+g \frac{\partial p_{n}}{\partial z_{n}}\right)_{z_{a}=H_{n}}=0
$$


where $\mathbf{g}$ is the gravitational acceleration.

At the interface of Layers $\mathrm{j}$ and $\mathrm{j}+1$, the boundary conditions are:

4. Continuity of the vertical acceleration, i.e.,

$$
-\left.\frac{1}{\rho_{j}} \frac{\partial p_{j}}{\partial z_{j}}\right|_{z_{j}=H_{j}}=-\left.\frac{1}{\rho_{j+1}} \frac{\partial p_{j+1}}{\partial z_{j+1}}\right|_{z_{j}=0}
$$

and

5. Continuity of the hydrodynamic pressure, i.e.,

$$
\left.p_{j}\right|_{z_{j}=H_{j}}=\left.p_{j+1}\right|_{z_{j: i}=0}
$$

Note that, strictly speaking, if the densities of the liquids in Layers $j$ and $j+1$ were indeed different, the hydrodynamic pressure would have a jump at the interface, and Eq. (8) would have to be replaced by [6]

$$
\left.\left(\frac{\partial^{2} p_{j}}{\partial t^{2}}+g \frac{\partial p_{j}}{\partial z_{j}}\right)\right|_{z_{j}=H_{j}}=\left.\left(\frac{\partial^{2} p_{j+1}}{\partial t^{2}}+g \frac{\partial p_{j+1}}{\partial z_{j+1}}\right)\right|_{z_{j+1}=0}
$$

However, the reason that $\rho_{j}$ is not equal to $\rho_{j+1}$ is derived from the mathematical modeling not from the original physical system. It should be noted that in the physical system the density of liquid is continuous at the interface. Also, if one uses Eq. (9) instead of Eq. (8) to solve the problem, one will obtain $\mathbf{n}$ distinct natural frequencies for each horizontal sloshing mode [6] which is believed to be unrealistic for the physical system considered. Therefore, Eq. (8) instead of Eq. (9) is used in this study.

The solutions for $\mathrm{p}_{\mathrm{j}}$ is expressed as the sum of the impulsive component and the convective component, i.e., 


$$
\mathrm{p}_{\mathrm{j}}=\mathrm{p}_{\mathrm{j}}{ }^{\mathrm{i}}+\mathrm{p}_{\mathrm{j}}^{\mathrm{c}}
$$

where the superscript i stands for the impulsive component and the superscript $\mathrm{c}$ stands for the convective component.

\section{A. Impulsive Component}

The function $p_{j}{ }^{i}$ must satisfy

$$
\nabla^{2} p_{j}{ }^{i}=0 \quad \text { for } \mathrm{j}=1,2 \ldots \mathrm{n}
$$

and the following boundary conditions:

$$
\begin{aligned}
& \left.\frac{\partial p_{1}^{i}}{\partial z_{1}}\right|_{z_{i}=0}=0 \\
& \left.\frac{\partial p_{j}^{i}}{\partial r}\right|_{r=R}=-\rho_{j} \ddot{x}(t) \cos \theta, \quad j=1,2 \ldots n \\
& \left.p_{n}^{i}\right|_{z_{i}=H_{i}}=0 \\
& -\left.\frac{1}{\rho_{j}} \frac{\partial p_{j}^{i}}{\partial z_{j}}\right|_{z_{j}=H_{j}}=-\left.\frac{1}{\rho_{j+1}} \frac{\partial p_{j+1}^{i}}{\partial z_{j+1}}\right|_{z_{j=1}=0} \\
& \left.p_{j}^{i}\right|_{z_{j}=H_{j}}=\left.p_{j=1}^{i}\right|_{z_{j=1}=0}
\end{aligned}
$$

The method of separation of variables is employed to solve Eq. (11), and the integration constants are determined from the boundary conditions. Satisfying Eqs. (11) and (12b), the function $\mathrm{p}_{\mathrm{j}}{ }^{\mathrm{i}}$ takes the form 


$$
\begin{aligned}
p_{j}^{i}= & {\left[-\frac{r}{R}+\sum_{k=1}^{\infty}\left(A_{j k} \cosh \left(\lambda_{k} \frac{z_{j}}{R}\right)+B_{j k} \sinh \left(\lambda_{k} \frac{z_{j}}{R}\right)\right)\right.} \\
& \left.\frac{J_{1}\left(\lambda_{k} \frac{r}{R}\right)}{J_{1}\left(\lambda_{k}\right)}\right] \rho_{j} R \ddot{x}(t) \cos \theta, \quad \text { for } j=1,2 \ldots n
\end{aligned}
$$

where $J_{1}$ is the Bessel function of the first kind of order $1, \lambda_{k}=$ the kth zero of $J_{i}^{\prime}$, the first derivative of $\mathrm{J}_{1}$, and $\mathrm{A}_{\mathrm{jk}}$ and $\mathrm{B}_{\mathrm{jk}}$ are the constants of integration that may be determined from the boundary conditions. From Eq. (12a), one obtains

$$
B_{1 \mathrm{k}}=0 \quad \text { for all } \mathrm{k}
$$

and from Eqs. (12d) and (12e), one obtains

$$
B_{(j+1) k}=\sinh \beta_{j k} A_{j k}+\cosh \beta_{j k} B_{j k}, \quad \text { for } j=1,2 \ldots n
$$

and

$$
\begin{aligned}
A_{(j+1) k} & =\frac{\rho_{j}}{\rho_{j+1}} \cosh \beta_{j k} A_{j k}+\frac{\rho_{j}}{\rho_{j+1}} \sinh \beta_{j k} B_{j k} \\
& +\left(1+\frac{\rho_{j}}{\rho_{j+1}}\right) \chi_{k}, \quad \text { for } j=1,2 \ldots n
\end{aligned}
$$

respectively. Equations (15a) and (15b) can be cast into he matrix form given by

$$
\begin{aligned}
\left\{\begin{array}{l}
A_{(j+1) k} \\
B_{(j+1) k}
\end{array}\right\} & =\left[\begin{array}{cc}
\frac{\rho_{j}}{\rho_{j+1}} \cosh \beta_{j k} & \frac{\rho_{j}}{\rho_{j+1}} \sinh \beta_{j k} \\
\sinh \beta_{j k} & \cosh \beta_{j k}
\end{array}\right]\left\{\begin{array}{c}
A_{j k} \\
B_{j k}
\end{array}\right\} \\
& +\left\{\begin{array}{c}
1-\frac{\rho_{j}}{\rho_{j+1}} \\
0
\end{array}\right\} x_{k}
\end{aligned}
$$


where $\beta_{j k}=\lambda_{\mathbf{k}} \mathrm{H}_{\mathrm{j}} / \mathrm{R}$ and

$$
x_{k}=\frac{2}{\lambda_{k}^{2}-1}
$$

To arrive at Eq. (15b), the following series expansion for $r / R$ is utilized.

$$
\frac{I}{R}=\sum_{k=1}^{\infty} \chi_{k} \frac{J_{1}\left(\lambda_{k} \frac{I}{R}\right)}{J_{1}\left(\lambda_{k}\right)}
$$

With the aid of the recursive relation defined by Eq. (15c) and the fact that $B_{1 k}=0$, the constants, $A_{n k}$ and $B_{n k}$, can be expressed in terms of the constant, $A_{1 k}$; then, $A_{1 k}$ can be determined from the following equation which is obtained from Eq. (12c).

$$
A_{n k} \cosh \beta_{n k}+B_{n k} \sinh \beta_{n k}=\chi_{k}
$$

After $A_{1 k}$ is obtained, the constants $A_{j k}$ and $B_{j k}$ for $j=2 \ldots n$ can be determined repeatedly by making use of Eq. (15c).

B. Convective Component

The convective component of the hydrodynamic pressure, $p_{j}{ }^{c}$, must satisfy

$$
\nabla^{2} \mathrm{p}_{\mathrm{j}}^{\mathrm{c}}=0 \quad \text { for } \mathrm{j}=1,2 \ldots \mathrm{n}
$$

and the following boundary conditions:

$$
\begin{aligned}
& \left.\frac{\partial p_{1}^{c}}{\partial z_{1}}\right|_{z_{1}=0}=0 \\
& \left.\frac{\partial p_{j}^{c}}{\partial r}\right|_{t=R}=0
\end{aligned}
$$




$$
\begin{aligned}
& \left.\left(\frac{\partial^{2} p_{n}^{c}}{\partial t^{2}}+g \frac{\partial p_{n}^{c}}{\partial z_{n}}\right)\right|_{z_{0}=H_{1}}=-\left.g \frac{\partial p_{n}^{i}}{\partial z_{n}}\right|_{z_{0}=H_{2}} \\
& -\left.\frac{1}{\rho_{j}} \frac{\partial p_{j}^{c}}{\partial z_{j}}\right|_{z_{1}=H_{j}}=-\left.\frac{1}{\rho_{j+1}} \frac{\partial p_{j+1}^{c}}{\partial z_{j+1}}\right|_{z_{j=1}=0}
\end{aligned}
$$

and

$$
\left.p_{j}^{c}\right|_{z_{j}=H_{j}}=\left.p_{j+1}^{c}\right|_{z_{j+1}=0}
$$

Following the same procedure used in solving Eq. (11), the function $p_{j}{ }^{c}$ that satisfies Eqs. (19) and (20b) takes the form

$$
\begin{aligned}
p_{j}^{c}= & \sum_{k=1}^{\infty}\left[C_{j k}(t) \cosh \left(\lambda_{k} \frac{z_{j}}{k}\right)+D_{j k}(t) \sinh \left(\lambda_{k} \frac{z_{j}}{R}\right)\right] \\
& \frac{J_{1}\left(\lambda_{k} \frac{r}{R}\right)}{J_{1}\left(\lambda_{k}\right)} \rho_{j} R \cos \theta
\end{aligned}
$$

in which $C_{j k}(t)$ and $D_{j k}(t)$ are the integration functions that may be determined by satisfying the boundary conditions. From Eq. (20a), one obtains

$$
D_{1 k}=0 \quad \text { for all } k
$$

and from Eqs. (20d) and (20e), one obtains

$$
\left\{\begin{array}{l}
C_{(j+1) k} \\
D_{(j+1) k}
\end{array}\right\}=\left[\begin{array}{cc}
\frac{\rho_{j}}{\rho_{j+1}} \cosh \beta_{j k} & \frac{\rho_{j}}{\rho_{j+1}} \sinh \beta_{j k} \\
\sinh \beta_{j k} & \cosh \beta_{j k}
\end{array}\right]\left\{\begin{array}{c}
C_{j k} \\
D_{j k}
\end{array}\right\}
$$


Similar to Eq. (15c), Eq. (23) defines a relation between the integration functions of jth layer and those of the $(j+1)$ th layer. By repeating application of Eq. (23) from $j=1$ to $j=n$ and making use of Eq. (22), one obtains the equation that links the $C_{1 k}$ with $C_{n k}$ and $D_{n k}$. Symbolically, this equation can be written as

$$
\left\{\begin{array}{c}
C_{n k} \\
D_{n k}
\end{array}\right\}=\left[\begin{array}{ll}
T_{11} & T_{12} \\
T_{21} & T_{22}
\end{array}\right]\left\{\begin{array}{c}
C_{1 k} \\
0
\end{array}\right\}
$$

where the $2 \times 2$ matrix in Eq. (24) is the transfer matrix, and $T_{11}, T_{12}, T_{21}$ and $T_{22}$ are its elements. Introducing a coefficient, $\eta_{n k}$ that represents the ratio of $D_{n k}$ to $C_{n k}$, i.e.,

$$
D_{n k}=\eta_{n k} C_{n k}
$$

from Eq. (24) $\eta_{\mathrm{nk}}$ is given by

$$
\eta_{n k}=\frac{T_{21}}{T_{11}}
$$

Finally, $C_{n k}$ may be determined by the following equation which is obtained from boundary condition Eq. (20c)

$$
\begin{aligned}
& \left(\cosh \beta_{n k}+\eta_{n k} \sinh \beta_{n k}\right) \ddot{C}_{n k}(t) \\
& +\frac{g \lambda_{k}}{R}\left(\sinh \beta_{n k}+\eta_{n k} \cosh \beta_{n k}\right) C_{n k}(t) \\
& =-\frac{g \lambda_{k}}{R}\left(A_{n k} \sinh \beta_{n k}+B_{n k} \cosh \beta_{n k}\right) \ddot{x}(t)
\end{aligned}
$$

The natural circular frequency for the kth vibration mode of the sloshing motion, denoted by $\omega_{k}$, can be obtained from the free vibration equation associated with Eq. (27), and the result is given by

$$
\omega_{\mathrm{k}}^{2}=\frac{\mathrm{g} \lambda_{\mathrm{k}}}{\mathrm{R}}\left(\frac{\eta_{\mathrm{nk}}+\tanh \beta_{\mathrm{nk}}}{1+\eta_{\mathrm{nk}} \tanh \beta_{\mathrm{nk}}}\right)
$$


where the coefficients $A_{n k}$ and $B_{n k}$ are obtained from the preceding section for the impulsive component. The solution to Eq. (28) is given by

$$
C_{n k}(t)=-\left(\frac{A_{n k} \tanh \beta_{n k}+B_{n k}}{\eta_{n k}+\tanh \beta_{n k}}\right) A_{k}(t)
$$

where $A_{k}(t)$ is the pseudoacceleration function for the kth vibration mode of the sloshing motion and is defined by

$$
A_{k}(t)=\omega_{k} \int_{0}^{t} \ddot{x}(t) \sin \left(\omega_{k}(t-\tau)\right) d \tau
$$

The convective component of the hydrodynamic pressure for the nth layer, $\mathrm{p}_{\mathrm{n}}{ }^{\mathrm{c}}$, is then obtained from Eq. (21) by setting $\mathrm{j}=\mathrm{n}$ and substituting Eqs. (25) and (30) into the resulting equation. The result is given by

$$
\begin{aligned}
p_{n}^{c}= & -\sum_{k=1}^{\infty}\left(\frac{A_{n k} \tanh \beta_{n k}+B_{n k}}{\eta_{n k}+\tanh \beta_{n k}}\right)\left(\cosh \lambda_{k} \frac{z_{n}}{R}+\eta_{n k} \sinh \lambda_{k} \frac{z_{n}}{R}\right) \\
& \frac{J_{1}\left(\lambda_{k} \frac{r}{R}\right)}{J_{1}\left(\lambda_{k}\right)} A_{k}(t) \rho_{n} R \cos \theta
\end{aligned}
$$

The sloshing wave height, $d(r, \theta, t)$, of an arbitrary point at the liquid surface is determined from

$$
\left.\mathrm{p}_{\mathrm{n}}^{c}\right|_{z_{\mathrm{n}}=\mathrm{H}_{\mathrm{a}}}=\rho_{\mathrm{n}} \mathrm{gd}(\mathrm{r}, \theta, \mathrm{t})
$$

and the result is expressed as

$$
d(r, \theta, t)=\left[\sum_{k=1}^{\infty} r_{k}\left(\frac{2}{\lambda_{k}^{2}-1}\right) \frac{A_{k}(t)}{g} \frac{J_{1}\left(\lambda_{k} \frac{r}{R}\right)}{J_{1}\left(\lambda_{k}\right)}\right] R \cos \theta
$$


in which $r_{k}$ is a dimensionless coefficient which is equal to one if the liquid has uniform density; therefore, $r_{k}$ represents the effect of the nonuniform liquid density on the kth mode of sloshing wave height. Also, another dimensionless coefficient, $\alpha_{k}$, is introduced to assess the effect of nonuniform liquid density on the sloshing frequencies; $\alpha_{k}$ is defined by rewriting Eq. (28) as

$$
\omega_{k}^{2}=\alpha_{k}\left(\frac{g \lambda_{k}}{R} \tanh \left(\lambda_{k} \frac{H}{R}\right)\right)
$$

Again, $\alpha_{k}$ has a value of one if the liquid has uniform density. Note that for the case of $n=2$ the closed form solutions for $r_{k}$ and $\alpha_{k}$ are available in Ref. 5. After $C_{n k}$ is obtained from Eq. (24), the coefficient $C_{1 k}$ may be computed by the equation given by

$$
C_{1 k}=\frac{1}{T_{11}} C_{n k}
$$

which is derived from Eq. (24). With the information for $D_{1 k}$ given in Eq. (22), the coefficients $C_{j k}$ and $D_{j k}$ for $j=2,3, \ldots, n$ can be calculated from Eq. (23) recursively, and the convective hydrodynamic pressure given by Eq. (21) can be computed as well. The maximum convective hydrodynamic pressure exerted on the tank wall is obtained by evaluating Eq. (21) at $r=R$, and $\theta=0$; the result can be cast into the following equation

$$
p^{c}(z, t)=\sum_{k=1}^{\infty} c_{k}(z) A_{k}(t) \rho_{b} R
$$

Note that in Eq. (36), the magnitude of the pressure is normalized by $\rho_{b}$ which is the maximum value of the liquid density.

\section{NUMERICAL RESULTS}

Unlike the case of tanks containing uniform liquid density where $H / R$ is the only parameter that controls the response, the parameters that control the response of tanks containing nonuniform liquid density are $H / R, \rho_{\mathfrak{r}} / \rho_{b}$, and the density variation along the liquid depth. For the numerical study presented herein, the variation of the liquid density between $\rho_{t}$ and $\rho_{b}$ is 
assumed to be either a linear or a cosine function. More precisely, if $\rho(z)$ represents the density at liquid depth of $z$, the linear function is given by

$$
\rho(z)=\rho_{t}+\left(\frac{H-z}{H}\right)\left(\rho_{b}-\rho_{t}\right)
$$

and the cosine function is given by

$$
\rho(z)=\rho_{t}+\left(\rho_{b}-\rho_{t}\right) \cos \left(\frac{\pi z}{2 H}\right)
$$

It is important to determine the number of layers needed to approximate the liquid field in order to get accurate results. Therefore, the study for the convergence of the natural frequency and sloshing displacement is performed first. Since the major contribution to the sloshing response comes from the fundamental mode of vibration, the convergence study for the frequency coefficient $\alpha_{k}$ and $r_{k}$ for $k=1$ is presented in Tables I and II, respectively, assuming either linear or cosine variation for the liquid density. In these tables, values of $\alpha_{1}$ and $r_{1}$ for tanks with $H / R$ $=0.5$ and 3 filled with liquid with $\rho_{\mathrm{l}} / \rho_{\mathrm{b}}=0.25,0.5$ and 0.75 were obtained by using different numbers of layers, and for simplicity the thicknesses of these layers are taken to be the same. As one can see from these tables that for larger value of $\rho_{\mathrm{t}} / \rho_{\mathrm{b}}$ and linear variation of density the convergence for $\alpha_{1}$ and $r_{1}$ is faster. Also, from the tables one may conclude that with $n=300$, the results are quite accurate. Thus all solutions presented hereinafter are obtained by using $\mathbf{n}$ $=300$. It should be mentioned herein that the approach presented in this report is implemented into a FORTRAN computer program that runs on PC 486 machine, and for $n=600$ the CPU time spent is less than 5 seconds.

The frequency coefficient $\alpha_{k}$ and sloshing displacement coefficient $r_{k}$ for $k=1$ are plotted in Figs. 3 and 4 , respectively, for linear and cosine variations of density as a function of $\rho_{\mathrm{l}} / \rho_{\mathrm{b}}$. Four different values of $\mathrm{H} / \mathrm{R}, 0.5,1,2$ and 3 , are considered. Examining the information presented in Figs. 3 and 4, one notices that the effects of nonuniform liquid density on the sloshing response are: (1) to decrease the fundamental natural frequency of the sloshing motion; and (2) to increase the sloshing wave height. This effect is more pronounced for broad tanks 
with low value of $\rho_{\mathrm{r}} / \rho_{\mathrm{b}}$, and these effects are not particularly sensitive to the detailed variation of the density value between $\rho_{t}$ and $\rho_{b}$. The increase of the sloshing wave height may be significant; for example, the value of $r_{1}$ is more than double for $H / R=0.5$ and $\rho_{t} / \rho_{b}<0.25$ compared with that for $H / R=0.5$ and $\rho_{\mathrm{t}} / \rho_{\mathrm{b}}=1$, the uniform density. To further confirm this finding, the maximum sloshing wave height of a liquid with $\rho_{\mathrm{t}} / \rho_{\mathrm{b}}=0.25$ in a tank of radius $R$ $=25 \mathrm{ft}(7.63 \mathrm{~m})$ and liquid height $\mathrm{H}=12.5 \mathrm{ft}(3.81 \mathrm{~m})$ subjected to the 29 -second horizontal component of the September 16, 1978 Tabas earthquake of Iran is computed. The peak acceleration of the Tabas recorded is denoted by $\ddot{x}_{g}$. The maximum sloshing wave height, denoted by $d_{\max }$, may be obtained by evaluating Eq. (33) at $r=R$ and $\theta=0$. For the first three modes of vibration, $d_{\max }$ may be expressed as

$$
d_{\max }=d_{1} \frac{A_{1}(t)}{g}+d_{2} \frac{A_{2}(t)}{g}+d_{3} \frac{A_{3}(t)}{g}
$$

in which the subscripts 1, 2 and 3 represent the first, second and third mode, respectively, and the coefficient $d_{i}, i=1,2$ and 3 has the unit of length. The first three natural frequencies, denoted by $f_{i}$, the value of $d_{i}$ and the maximum values of $A_{i}(t)$, denoted by $S A_{i}$, for $i=1,2$ and 3 are tabulated in Table III for the cases of linear, consine and uniform liquid density variations. Also, listed in the Table III are the $d_{\max }$ obtained from Eq. (39) by the square root of the sum of the squares (SRSS) of the three components. One, again, can see clearly that the sloshing wave height increases from the uniform density case to the linear variation case by $67 \%$, and for the cosine variation case the increase is $46 \%$.

The distribution function of the convective hydrodynamic pressure exerted on the tank wall, $c_{k}(z), k=1$, is plotted in Figs. 5 and 6 for $H / R=0.5$ and 3, respectively, for the values of $\rho_{\mathrm{r}} / \rho_{\mathrm{b}}=0.25$ and 0.75 for the linear and cosine variations of liquid density. Plotted in the same figures and shown by the dotted lines are the corresponding information for the case of uniform liquid density of $\rho_{b}$. It is clearly shown in these two figures that the convective hydrodynamic pressure may be far off the true solution if uniform liquid density is assumed. It is noted herein that the accuracy of the pressure computed has been checked against those presented in Ref. 5 for the case of $n=2$. 


\section{CONCLUSIONS}

A study on the effect of nonuniform liquid density on the sloshing response of an upright circular cylindrical tank is presented. The response quantities examined include the natural sloshing frequencies, surface sloshing displacement, and convective hydrodynamic pressure. It is shown that nonuniform liquid density has two main effects on the sloshing response: (1) increasing of the sloshing wave height; and (2) decreasing of the sloshing frequencies. The magnitude of the convective hydrodynamic pressure induced in the nonuniform liquid density may be quite different from that of uniform liquid. In the numerical example it is further shown that if the effect of the nonuniform liquid density is considered, the maximum sloshing wave height may be increased by more than 50 percent when compared with that of the identical tank filled with uniform liquid.

\section{ACKNOWLEDGMENT}

This work was supported by the U.S. Department of Energy, Technology Support Programs under Contract W-31-109-Eng-38.

\section{REFERENCES}

1. G. W. Housner, "Dynamic Pressure on Accelerated Fluid Container," Bull. Seismological Soc. of Am., Vol. 47, No. 1, pp. 15-35 (1957).

2. M. F. Bauer, "Fluid Oscillations in the Containers of a Space Vehicle and Their Influence Upon Stability," NASA Report TR.R-187 (1964).

3. J. Y. Yang, "Dynamic Behavior of Fluid-Tank Systems," Ph.D. thesis, Rice University, Houston, TX (1976).

4. K. K. Bandyopadhyay, "Overview of Seismic Panel Activities," Proc. 3rd DOE Natural Phenomena Hazards Mitigation Conf., U.S. Dept. of Energy, Washington, DC, pp. 423429 (1991).

5. Y. Tang, "Dynamic Response of Tank Containing Two Liquids," J. Engrg. Mech. Div., ASCE, Vol. 119, No. 3, pp. $531-548$ (1993). 
6. Y. Tang and Y. W. Chang, "The Exact Solutions to the Dynamic Response of Tanks Containing Two Liquids," Argonne National Laboratory Report ANL/RE-93/2 (1993).

7. A. S. Veletsos, "Seismic Response and Design of Liquid Storage Tanks," Guidelines for the Seismic Design of Oil and Gas Pipeline Systems, ASCE, New York, NY, pp. 255-370 and 443-461 (1984).

8. M. A. Haroun and G. W. Housner, "Complications in Free Vibration Analysis of Tanks," J. Engrg. Mech. Div., ASCE, Vol. 108, No. 5, pp. 801-818 (1981). 
Table I. Convergence Table of Sloshing Frequency Coefficient, $\alpha_{1}$

\begin{tabular}{|c|c|c|c|c|c|c|}
\hline \multirow{2}{*}{$\begin{array}{l}\text { No. of } \\
\text { Layers }\end{array}$} & \multicolumn{2}{|c|}{$\rho_{\mathrm{t}} / \rho_{\mathrm{b}}=0.75$} & \multicolumn{2}{|c|}{$\rho_{\mathrm{r}} / \rho_{\mathrm{b}}=0.50$} & \multicolumn{2}{|c|}{$\rho_{\mathrm{t}} / \rho_{\mathrm{b}}=0.25$} \\
\hline & Linear & Cosine & Linear & Cosine & Linear & Cosine \\
\hline \multicolumn{7}{|c|}{$\mathrm{H} / \mathrm{R}=0.5$} \\
\hline 2 & 0.976 & 0.975 & 0.947 & 0.948 & 0.911 & 0.917 \\
\hline 5 & 0.957 & 0.949 & 0.899 & 0.884 & 0.813 & 0.797 \\
\hline 10 & 0.950 & 0.938 & 0.880 & 0.856 & 0.769 & 0.738 \\
\hline 50 & 0.944 & 0.928 & 0.864 & 0.832 & 0.729 & 0.682 \\
\hline 100 & 0.943 & 0.927 & 0.862 & 0.829 & 0.724 & 0.674 \\
\hline 150 & 0.943 & 0.927 & 0.861 & 0.827 & 0.722 & 0.672 \\
\hline 200 & 0.943 & 0.927 & 0.861 & 0.827 & 0.721 & 0.670 \\
\hline 250 & 0.943 & 0.927 & 0.861 & 0.827 & 0.721 & 0.670 \\
\hline 300 & 0.943 & 0.926 & 0.860 & 0.826 & 0.720 & 0.669 \\
\hline 600 & 0.943 & 0.926 & 0.860 & 0.826 & 0.720 & 0.668 \\
\hline \multicolumn{7}{|c|}{$\mathrm{H} / \mathrm{R}=3.0$} \\
\hline 2 & 1.000 & 1.000 & 0.999 & 0.999 & 0.999 & 0.999 \\
\hline 5 & 0.996 & 0.995 & 0.990 & 0.987 & 0.978 & 0.973 \\
\hline 10 & 0.992 & 0.988 & 0.979 & 0.970 & 0.947 & 0.933 \\
\hline 50 & 0.987 & 0.980 & 0.964 & 0.957 & 0.909 & 0.874 \\
\hline 100 & 0.986 & 0.979 & 0.962 & 0.944 & 0.902 & 0.864 \\
\hline 150 & 0.986 & 0.979 & 0.961 & 0.942 & 0.900 & 0.862 \\
\hline 200 & 0.986 & 0.979 & 0.960 & 0.942 & 0.899 & 0.860 \\
\hline 250 & 0.986 & 0.978 & 0.960 & 0.941 & 0.898 & 0.859 \\
\hline 300 & 0.986 & 0.978 & 0.960 & 0.941 & 0.898 & 0.858 \\
\hline 600 & 0.986 & 0.978 & 0.960 & 0.940 & 0.897 & 0.857 \\
\hline
\end{tabular}


Table II. Convergence Table of Sloshing Displacement Coefficient, $r_{1}$

\begin{tabular}{|c|c|c|c|c|c|c|}
\hline \multirow{3}{*}{$\begin{array}{l}\text { No. of } \\
\text { Layers }\end{array}$} & \multicolumn{6}{|c|}{ Displacement } \\
\hline & \multicolumn{2}{|c|}{$\rho_{d} / \rho_{b}=0.75$} & \multicolumn{2}{|c|}{$\rho_{\mathrm{d}} / \rho_{\mathrm{b}}=0.50$} & \multicolumn{2}{|c|}{$\rho_{\mathrm{l}} / \rho_{\mathrm{b}}=0.25$} \\
\hline & Linear & Cosine & Linear & Cosine & Linear & Cosine \\
\hline & \multicolumn{6}{|c|}{$\mathrm{H} / \mathrm{R}=0.5$} \\
\hline 2 & 1.064 & 1.067 & 1.150 & 1.148 & 1.271 & 1.248 \\
\hline 5 & 1.112 & 1.134 & 1.290 & 1.337 & 1.628 & 1.689 \\
\hline 10 & 1.130 & 1.162 & 1.348 & 1.428 & 1.825 & 1.979 \\
\hline 50 & 1.145 & 1.185 & 1.401 & 1.514 & 2.031 & 2.322 \\
\hline 100 & 1.147 & 1.188 & 1.408 & 1.526 & 2.061 & 2.375 \\
\hline 150 & 1.147 & 1.190 & 1.410 & 1.530 & 2.071 & 2.393 \\
\hline 200 & 1.147 & 1.190 & 1.411 & 1.532 & 2.076 & 2.402 \\
\hline 250 & 1.148 & 1.190 & 1.412 & 1.533 & 2.079 & 2.408 \\
\hline 300 & 1.148 & 1.911 & 1.413 & 1.534 & 2.081 & 2.411 \\
\hline 600 & 1.148 & 1.191 & 1.414 & 1.536 & 2.087 & 2.421 \\
\hline \multicolumn{7}{|c|}{$\mathrm{H} / \mathrm{R}=3.0$} \\
\hline 2 & 1.009 & 1.009 & 1.021 & 1.021 & 1.038 & 1.035 \\
\hline 5 & 1.030 & 1.040 & 1.079 & 1.101 & 1.174 & 1.203 \\
\hline 10 & 1.042 & 1.060 & 1.116 & 1.158 & 1.282 & 1.360 \\
\hline 50 & 1.055 & 1.079 & 1.156 & 1.222 & 1.415 & 1.576 \\
\hline 100 & 1.057 & 1.082 & 1.161 & 1.231 & 1.435 & 1.611 \\
\hline 150 & 1.057 & 1.083 & 1.163 & 1.235 & 1.442 & 1.623 \\
\hline 200 & 1.057 & 1.083 & 1.164 & 1.236 & 1.446 & 1.629 \\
\hline 250 & 1.058 & 1.084 & 1.165 & 1.237 & 1.448 & 1.633 \\
\hline 300 & 1.058 & 1.084 & 1.165 & 1.238 & 1.449 & 1.636 \\
\hline 600 & 1.058 & 1.084 & 1.166 & 1.239 & 1.453 & 1.642 \\
\hline
\end{tabular}


Table III. Comparison of Response Quantities of the Tank-Liquid Systems Considered in Numerical Example

\begin{tabular}{|c|c|c|c|}
\hline & Linear & Cosine & Uniform \\
\hline $\mathrm{f}_{1}(\mathrm{~Hz})$ & 0.151 & 0.140 & 0.209 \\
\hline $\mathrm{f}_{2}(\mathrm{~Hz})$ & 0.343 & 0.322 & 0.415 \\
\hline $\mathrm{f}_{3}(\mathrm{~Hz})$ & 0.462 & 0.438 & 0.528 \\
\hline$d_{1} / \mathbf{R}$ & 1.742 & 2.018 & 0.837 \\
\hline $\mathrm{d}_{2} / \mathbf{R}$ & 0.09 & 0.148 & 0.07 \\
\hline $\mathrm{d}_{3} / \mathrm{R}$ & 0.04 & 0.05 & 0.03 \\
\hline$\frac{S A_{1}}{g}$ & 0.212 & 0.157 & 0.265 \\
\hline$\frac{\mathrm{SA}_{2}}{\mathrm{~g}}$ & 0.523 & 0.534 & 0.442 \\
\hline$\frac{\mathrm{SA}_{3}}{\mathrm{~g}}$ & 0.574 & 0.471 & 0.769 \\
\hline$\frac{d_{\max }}{R} \cdot\left(\frac{g}{\ddot{x}_{g}}\right)$ & 0.376 & 0.331 & 0.225 \\
\hline
\end{tabular}




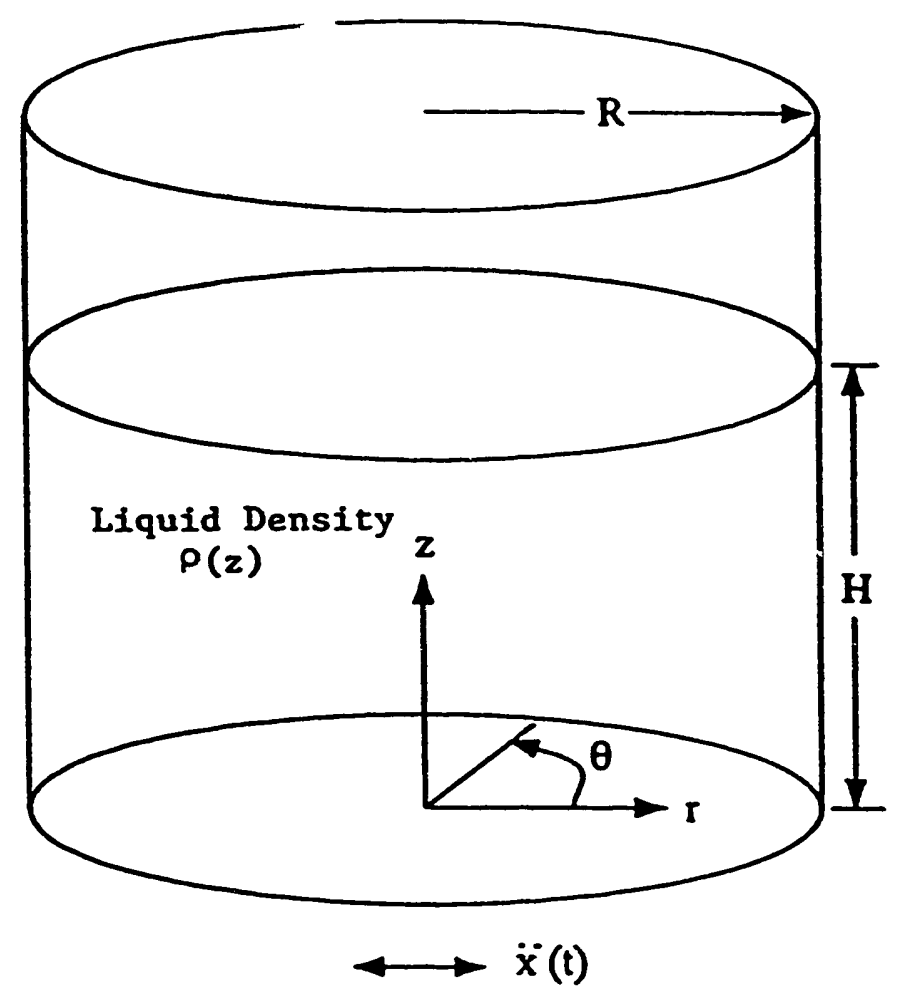

Fig. 1. System Considered 


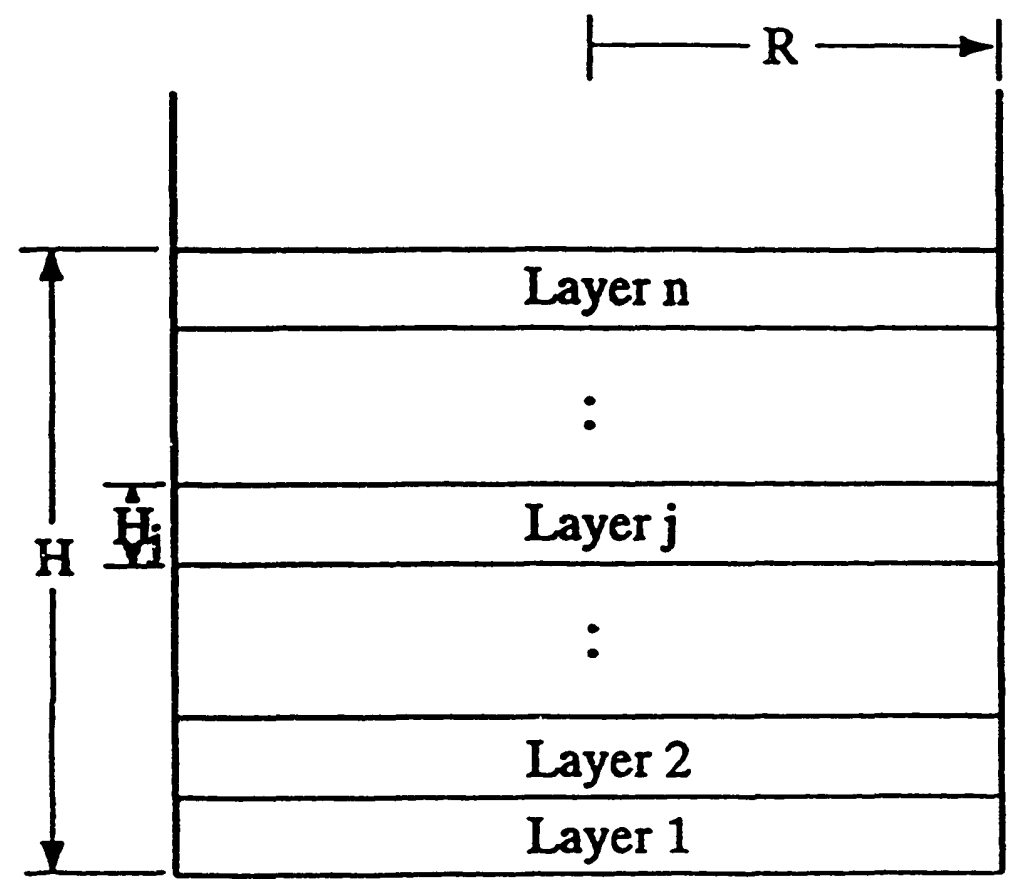

Fig. 2. Mathematical Model for the Liquid Field 

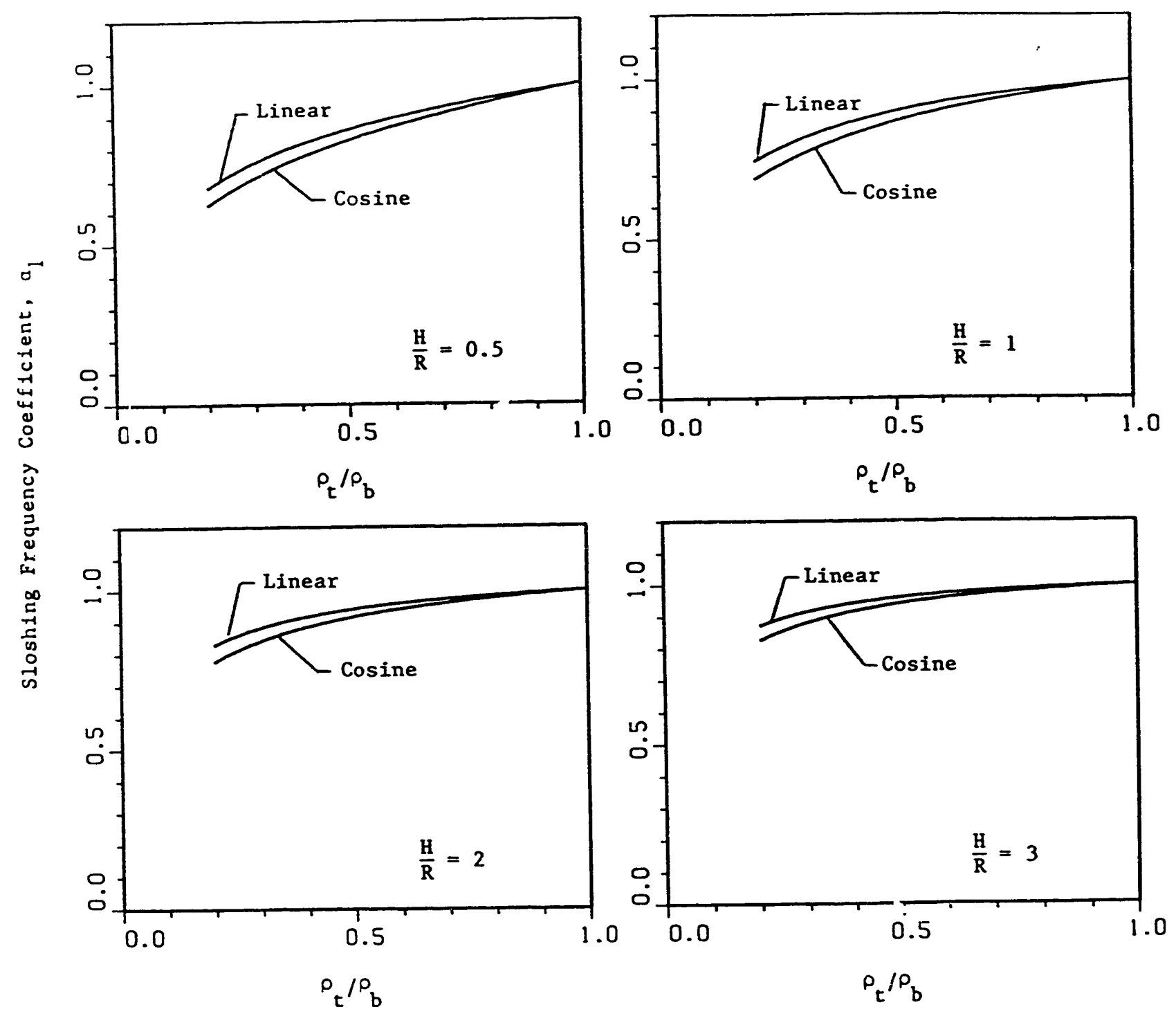

Fig. 3. Effect of Liquid Density on the Sloshing Frequency 

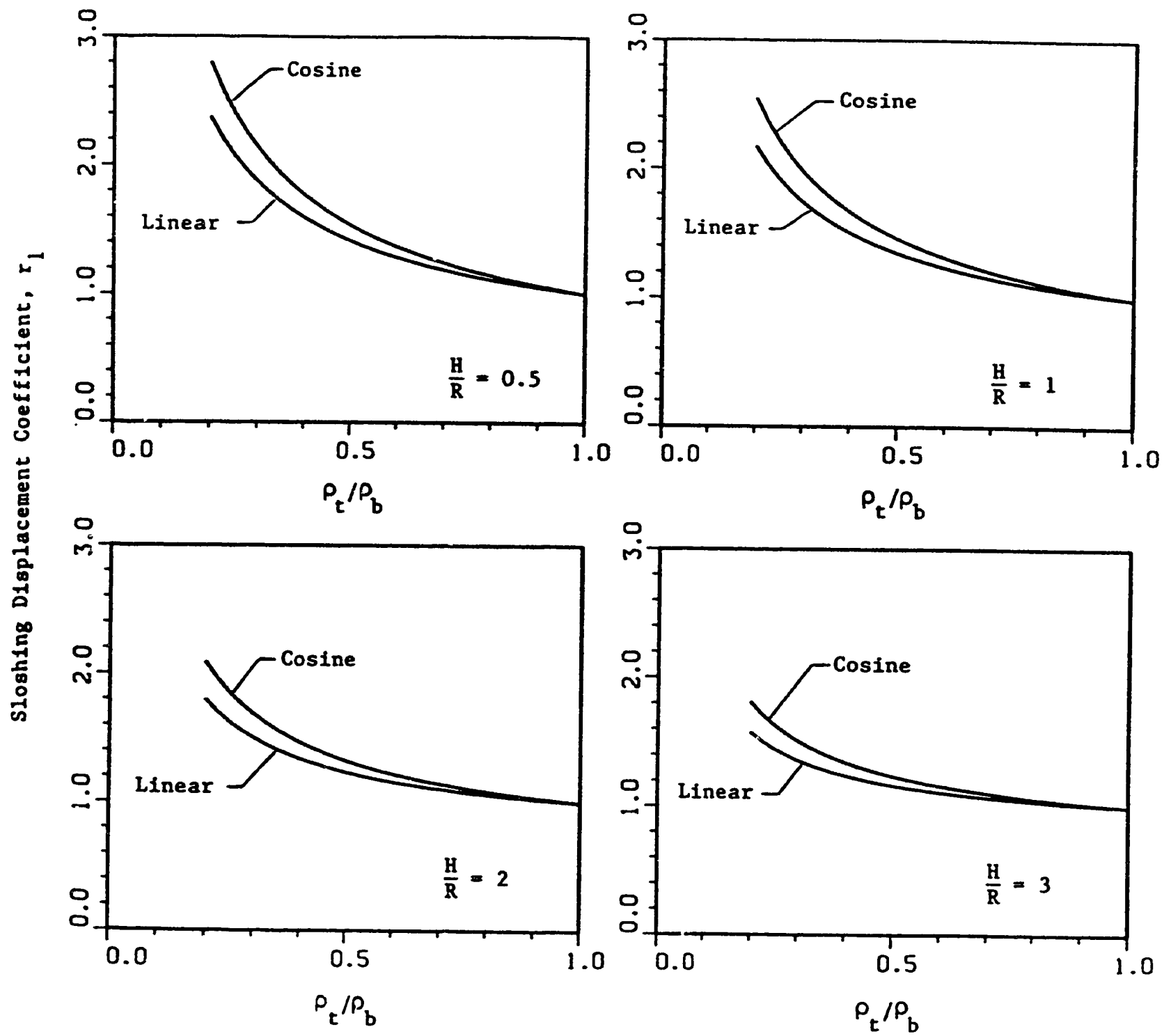

Fig. 4. Effect of Liquid Density on the Sloshing Displacement 


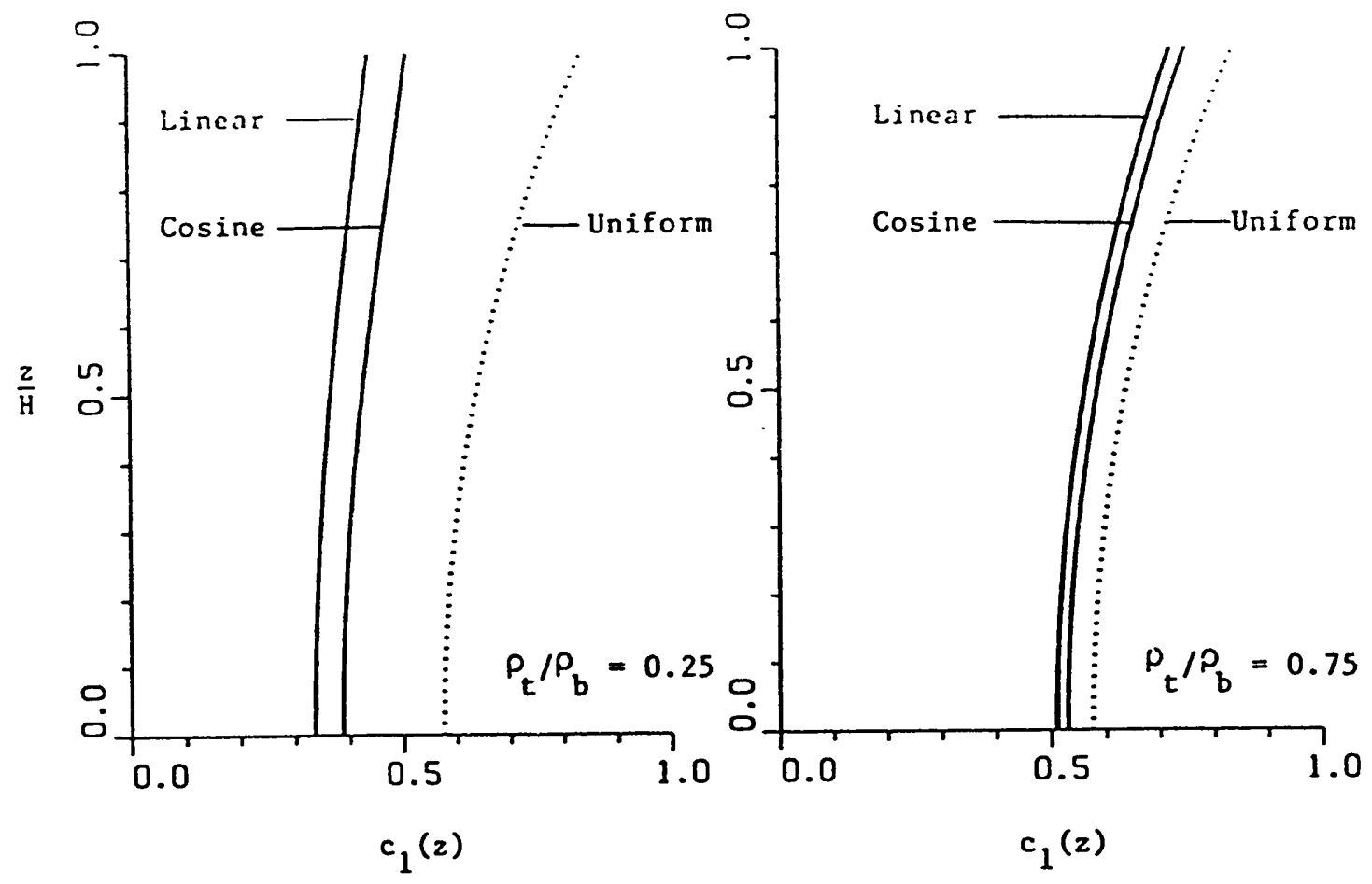

Fig. 5. Convective Pressure Exerted on Wall of Rigid Tanks with $H / R=0.5$
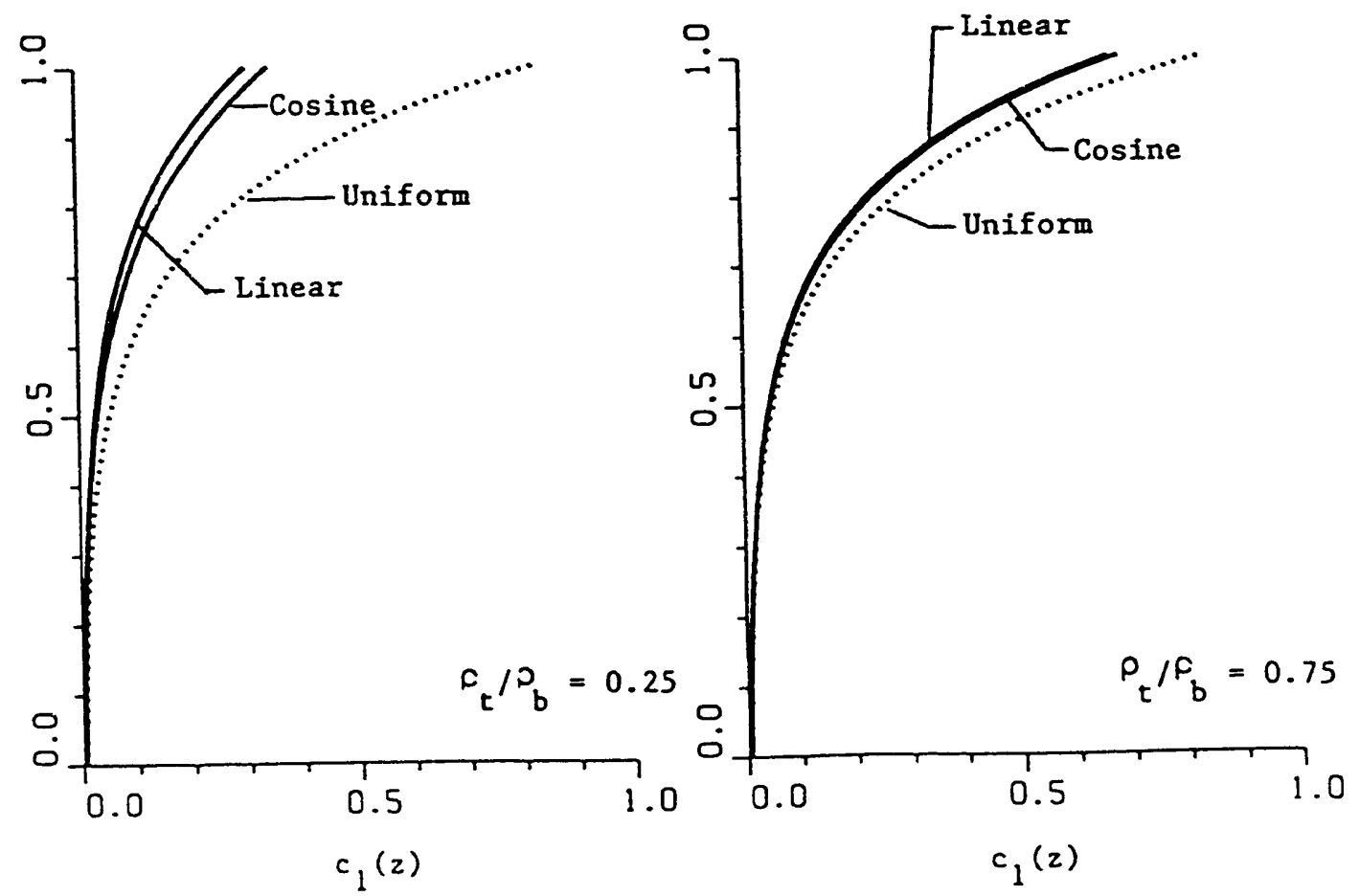

Fig. 6. Convective Pressure Exerted on Wall of Rigid Tanks with $H / R=3$ 

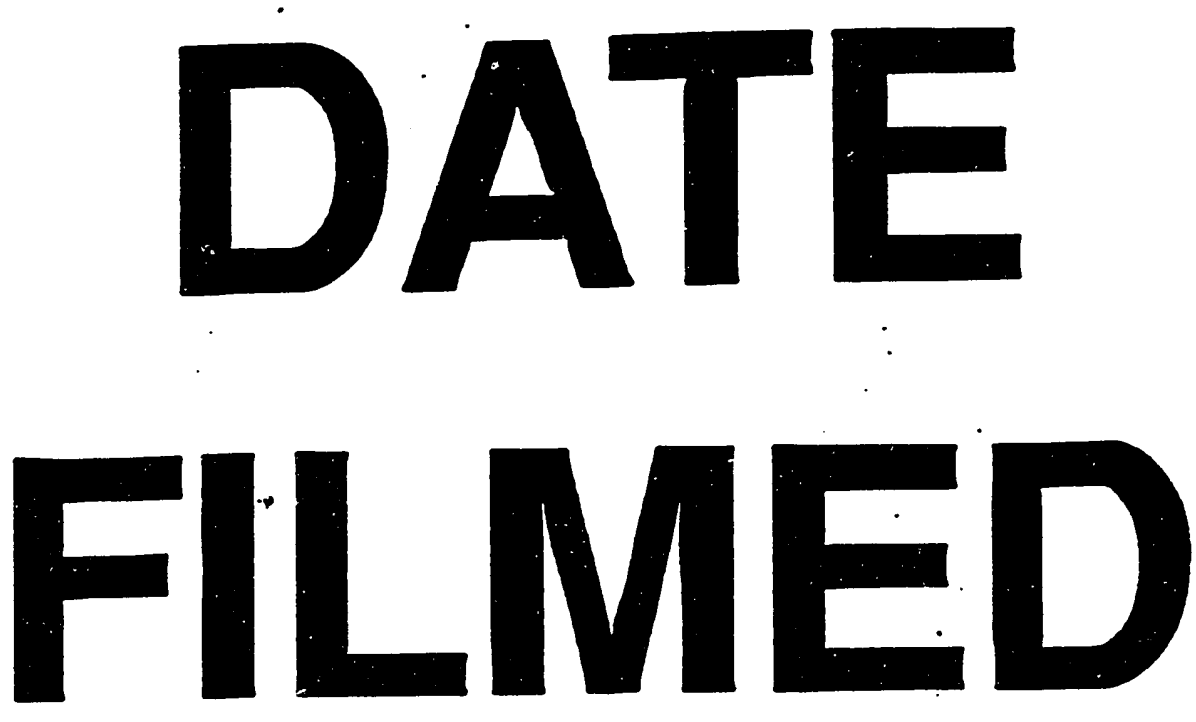

$1 / 5 / 94$
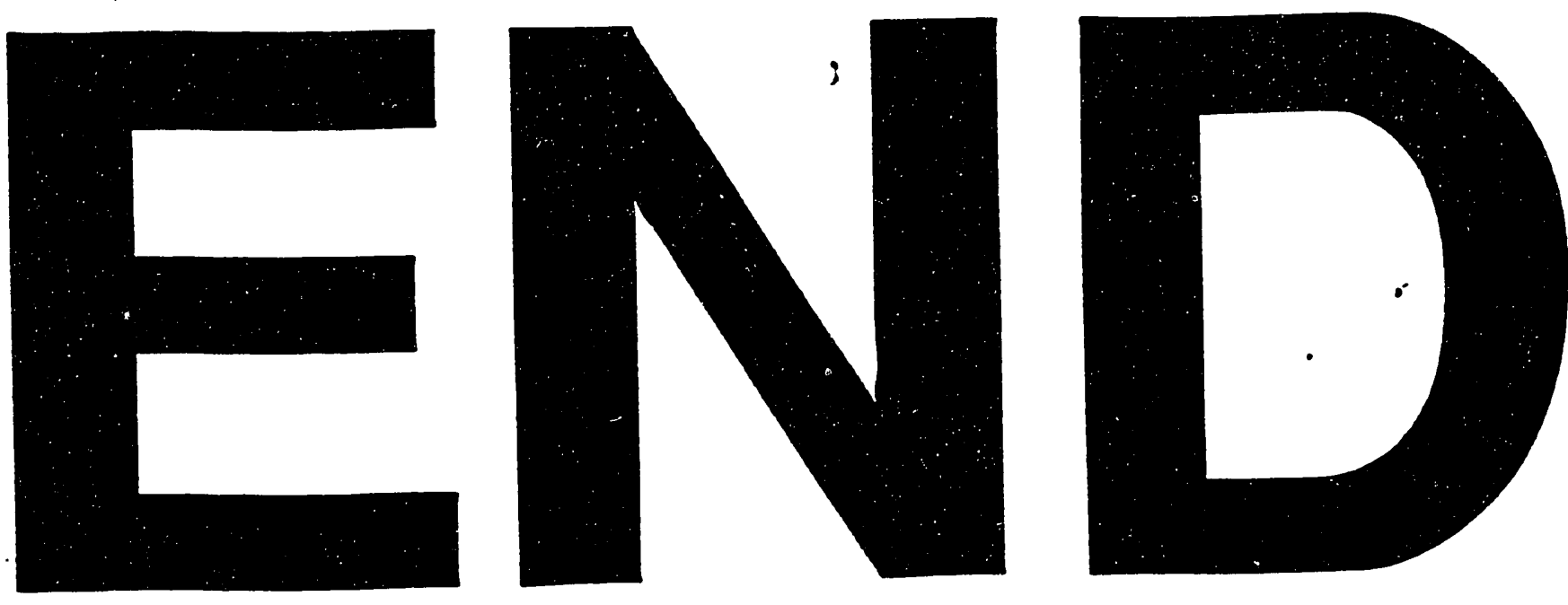